\title{
THE RECTIFIABLE METRIC ON THE SET OF CLOSED SUBSPACES OF HILBERT SPACE
}

\author{
LAWRENCE G. BROWN
}

\begin{abstract}
Consider the set of selfadjoint projections on a fixed Hilbert space. It is well known that the connected components, under the norm topology, are the sets $\{p: \operatorname{rank} p=\alpha, \operatorname{rank}(1-p)=\beta\}$, where $\alpha$ and $\beta$ are appropriate cardinal numbers. On a given component, instead of using the metric induced by the norm, we can use the rectifiable metric $d_{r}$ which is defined in terms of the lengths of rectifiable paths or, equivalently in this case, the lengths of $\varepsilon$-chains. If $\|p-q\|<1$, then $d_{r}(p, q)=\sin ^{-1}(\|p-q\|)$, but if $\|p-q\|=1$, $d_{r}(p, q)$ can have any value in $\left[\frac{\pi}{2}, \pi\right]$ (assuming $\alpha$ and $\beta$ are infinite). If $d_{r}(p, q) \neq \frac{\pi}{2}$, a minimizing path joining $p$ and $q$ exists; but if $d_{r}(p, q)=\frac{\pi}{2}$, a minimizing path exists if and only if $\operatorname{rank}(p \wedge(1-q))=\operatorname{rank}(q \wedge(1-p))$.
\end{abstract}

\section{INTRODUCTION}

Let $H$ be a Hilbert space, possibly nonseparable, and let $\mathscr{P}$ be the set of (selfadjoint) projections on $H$. For $p, q$ in $\mathscr{P}$, let $d_{n}(p, q)=\|p-q\|$, where " \|\| " is operator norm. Since $\|p-q\| \leq 1$, we can also define $d_{a}(p, q)=$ $\sin ^{-1}(\|p-q\|)$. So $d_{n}$ is the metric induced by the norm, and $d_{a}$ is the "angular metric." The fact that $d_{a}$ is a metric will be proved later (possibly this is folklore).

If $\alpha$ and $\beta$ are cardinal numbers such that $\alpha+\beta=\operatorname{dim} H$ (orthogonal dimension), let $C_{\alpha, \beta}=\{p \in \mathscr{P}: \operatorname{rank} p=\alpha, \operatorname{rank}(1-p)=\beta\}$. Then the various $C_{\alpha, \beta}$ 's are the connected components of $\mathscr{P}$. It is well known that $\|p-q\|<1$ implies that $p$ and $q$ are in the same component.

Some of our study will rely on the availability of a classification, up to unitary equivalence, of pairs of projections. This was first given by Dixmier [6] and Krein, Krasnosel'skiī, and Mil'man [9], independently. The note on p. 18 of [5] and the introduction to [16] give other historical information and references for this subject. Some other references are $[4,8,10, \S 3$, and 12].

If $p$ and $q$ are projections with ranges $M$ and $N$, let $H_{11}=M \cap N, H_{10}=$ $M \cap N^{\perp}, H_{01}=M^{\perp} \cap N, H_{00}=M^{\perp} \cap N^{\perp}$ and $H_{0}=\left(H_{11} \oplus H_{10} \oplus H_{01} \oplus H_{00}\right)^{\perp}$. If $H_{0}=H, M$ and $N$ are said to be in generic position ([8], Dixmier uses the term "position $p$ "). We will also refer to $H_{0}$ as the generic part of $H, M \cap H_{0}$ as the generic part of $M$, etc.

The basic example of two subspaces in generic position occurs when $\operatorname{dim} H=$

Received by the editors September 10,1990 and, in revised form, February 1, 1991.

1980 Mathematics Subject Classification (1985 Revision). Primary 46C99, 47A99, 47C15.

Key words and phrases. Hilbert space, projection, rectifiable. 
2, $\operatorname{dim} M=\operatorname{dim} N=1$, and $M$ and $N$ have angle $\theta$, where $0<\theta<\frac{\pi}{2}$. The most general generic pair is a direct integral of such two-dimensional examples, for various values of $\theta$. One way to describe an arbitrary pair of projections more explicitly is as follows:

It is possible to identify both $H_{0} \cap M$ and $H_{0} \cap M^{\perp}$, with $L^{2}(X)$, for some measure space $X$, in such a way that the generic parts of $p$ and $q$ are given by

$$
p_{0}=\left[\begin{array}{ll}
1 & 0 \\
0 & 0
\end{array}\right] \text { and } q_{0}=\left[\begin{array}{cc}
\cos ^{2} \varphi & \cos \varphi \sin \varphi \\
\cos \varphi \sin \varphi & \sin ^{2} \varphi
\end{array}\right],
$$

where $\varphi$ is a measurable function on $X$ such that $0<\varphi(x)<\frac{\pi}{2}, \forall x$. Here $p_{0}$ and $q_{0}$ operate on $L^{2}(X) \oplus L^{2}(X)$ and the matrices are operator matrices whose entries are multiplication operators.

In Theorem 12 below we use another method of describing generic pairs of subspaces, given by Halmos [8]. He shows that $N$ is the graph of a suitable (in general unbounded) operator $T: M \rightarrow M^{\perp}$. By using the polar decomposition of $T$, we can identify $M$ with $M^{\perp}$ in such a way that $T$ is positive and selfadjoint (this is part of Halmos' argument). In the description given above $T$ is the multiplication operator on $L^{2}(X)$ induced by $\tan \varphi$. The idea of representing $N$ as a graph can actually be used in some nongeneric cases also. It can be used whenever $H_{01}=0$, provided we weaken the requirements for $T$ sufficiently.

When we use the classification theory for pairs of projections below, we will often refer to the "canonical representation of the pair $(p, q)$ ", though the use of the term "canonical" is not strictly correct. The pair consisting of the Hilbert space $L^{2}(X)$ and the multiplication operator induced by $\varphi$ is uniquely determined up to unitary equivalence, but $X$ itself is not uniquely determined.

We now discuss the basic concepts relating to rectifiable metrics. This discussion will be oversimplified because certain difficulties which could arise in general do not occur in $\mathscr{P}$. Further information on this is contained in Chapter $1, \S \mathrm{B}$ of [7] and Chapters 3,4 of [13]. Let $(X, d)$ be a metric space. The concepts of rectifiable path and arc length of a path are defined for paths in $X$ in exactly the same way as for paths in $\mathbf{R}^{n}$. If two points $x$ and $y$ can be joined by a rectifiable path, we define $d_{r}(x, y)$ to be the infimum of the lengths of rectifiable paths joining $x$ and $y$. Clearly $d_{r}$ is a metric if it is everywhere defined, and $d_{r} \geq d$. The metric $d$ could be called "rectifiable" if $d_{r}=d$. Any $d$-rectifiable path is also $d_{r}$-rectifiable and has the same arc lengths relative to $d_{r}$ and $d$. (Thus $d_{r}$, if everywhere defined, is a rectifiable metric, not necessarily equivalent to $d$.) A minimizing path joining $x$ and $y$ is a rectifiable arc, parametrized by arc length, whose length is exactly $d_{r}(x, y)$. Clearly if a rectifiable path joining $x$ and $y$ of length $d_{r}(x, y)$ exists, then a minimizing path also exists.

If $\varepsilon>0$, an $\varepsilon$-chain joining $x$ and $y$ is a finite sequence of points in $X$, $x=x_{0}, x_{1}, x_{2}, \ldots, x_{n}=y$, such that $d\left(x_{i-1}, x_{i}\right) \leq \varepsilon$ for $1 \leq i \leq n$. The length of this $\varepsilon$-chain is $\sum_{1}^{n} d\left(x_{i-1}, x_{i}\right)$. It is well known that any two points in the same component can be joined by an $\varepsilon$-chain. For such $x, y$ let $d^{\varepsilon}(x, y)$ be the infimum of the lengths of $\varepsilon$-chains joining $x$ and $y$. Clearly $d^{\varepsilon}$ is a metric on each component of $X, d^{\varepsilon} \geq d$ (also $d^{\varepsilon}$ is equivalent to $d$ ), and $\varepsilon_{1}<\varepsilon_{2} \Rightarrow d^{\varepsilon_{1}} \geq d^{\varepsilon_{2}}$. Let $d^{\prime}(x, y)=\lim _{\varepsilon \rightarrow 0^{+}} d^{\varepsilon}(x, y)$. $d^{\prime}$ may take the value 
$\infty$, but it clearly satisfies the triangle inequality. Thus $d^{\prime}$ is a metric (no longer necessarily equivalent to $d$ ) if finite-valued. Clearly $d^{\prime} \leq d_{r}$, wherever $d_{r}$ is defined. In our case $d^{\prime}=d_{r}$ and they are equivalent to $d_{n}$ on each component $C_{\alpha, \beta}$. (In general a different concept of rectifiable can be defined by saying $d$ is "rectifiable" if $d=d^{\prime}$, and it follows from a result in [7] that this concept is equivalent if $(X, d)$ is complete, but presumably in general $\left(d^{\prime}\right)^{\prime}$ may be unequal to $d^{\prime}$. The only general theorems the author has heard of giving the existence of rectifiable paths between two points such that $d^{\prime}(x, y)<\infty$ have compactness hypotheses. However, the author is not an expert.)

Finally, we point out that if $H$ is finite dimensional, the concept of minimizing path used in this paper does not coincide with the usual geodesics on Grassmannians, as studied in differential geometry. The reason is that the differential-geometric geodesics, defined in terms of a Riemannian metric, are related to the Hilbert-Schmidt norm rather than the operator norm. Our minimizing paths do coincide with the differential-geometric geodesics if $\alpha$ or $\beta$ is 1 (or 0 ). The reason is that for rank 2 selfadjoint operators of trace 0 the Hilbert-Schmidt norm is proportional to the operator norm.

We gratefully acknowledge that we learned about rectifiable metrics in a differential geometry course taught by H. Gluck, probably in 1967-1968. Our notation may be different. We are also grateful for bibliographical help from P. de la Harpe, G. Pedersen, N. C. Phillips, and N. Salinas.

After this paper was written we learned of some related work by other authors. This will be discussed in Remark 5 of $\S 3$.

\section{MAIN Results}

The next two elementary lemmas are stated only for reference.

Lemma 1. Assume $\theta_{1}$ and $\theta_{2}$ are nonnegative numbers such that $\theta_{1}+\theta_{2} \leq$ $\pi$ and $u, v, w$ are unit vectors in $H$ such that $\operatorname{Re}(u, v)=\cos \theta_{1}$ and $\operatorname{Re}(v, w)=\cos \theta_{2}$. Then $\operatorname{Re}(u, w) \geq \cos \left(\theta_{1}+\theta_{2}\right)$.

Lemma 2. Assume $u$ is a unit vector in $H$ and $M$ a closed subspace of $H$. Let $\theta$ denote the minimum of angles between $u$ and unit vectors $v$ in $M$ (take $\theta=\frac{\pi}{2}$ if $\left.M=\{0\}\right)$. Then $\operatorname{dist}(u, M)=\sin \theta$.

Lemma 3. Assume $\theta_{1}$ and $\theta_{2}$ are nonnegative numbers such that $\theta_{1}+\theta_{2}<\frac{\pi}{2}$ and $p, q, r$ are in $\mathscr{P}$ such that $\|p-q\|=\sin \theta_{1}$ and $\|q-r\|=\sin \theta_{2}$. Then $\|p-r\| \leq \sin \left(\theta_{1}+\theta_{2}\right)$.

Proof. Let $L, M, N$ be the ranges of $p, q, r$. It is known that $\|p-r\|$ (for example) is the larger of $\sup \{\operatorname{dist}(u, N): u$ is a unit vector in $L\}$ and $\sup \{\operatorname{dist}(w, L): w$ is a unit vector in $N\}$. Let $u$ be a unit vector in $L$. Since $\operatorname{dist}(u, M) \leq \sin \theta_{1}$, there is a unit vector $v$ in $M$ such that $\operatorname{Re}(u, v) \geq \cos \theta_{1}$ by Lemma 2 . Since $\operatorname{dist}(v, N) \leq \sin \theta_{2}$, there is a unit vector $w$ in $N$ such that $\operatorname{Re}(v, w) \geq \cos \theta_{2}$ by Lemma 2 . Then by Lemma $1, \operatorname{Re}(u, w) \geq \cos \left(\theta_{1}+\theta_{2}\right)$. Thus, again by Lemma $2, \operatorname{dist}(u, N) \leq \sin \left(\theta_{1}+\theta_{2}\right)$. Similarly, the second supremum is at most $\sin \left(\theta_{1}+\theta_{2}\right)$.

Remark. Unless $\theta_{1}$ or $\theta_{2}$ is $0, \sin \left(\theta_{1}+\theta_{2}\right)<\sin \theta_{1}+\sin \theta_{2}$. Thus the conclusion of Lemma 3 is stronger than the triangle inequality for \|\| .

Corollary 4. $d_{a}$ satisfies the triangle inequality. Hence $d_{a}$ is a metric on $\mathscr{P}$, equivalent to $d_{n}$. 
Proof. Assume $p, q, r$ are in $\mathscr{P}$ and $d_{a}(p, q)=\theta_{1}, d_{a}(q, r)=\theta_{2}$. For the proof that $d_{a}(p, r) \leq \theta_{1}+\theta_{2}$ it is clearly permissible to assume that $\theta_{1}+\theta_{2}<\frac{\pi}{2}$. Thus Lemma 3 applies.

Remark. It was already known how to calculate $\|p-q\|$ in terms of the canonical representation of the pair $(p, q)$. Using this, we see that $d_{a}(p, q)=\frac{\pi}{2}$ if $H_{01}$ or $H_{10}$ is not $\{0\}$ and otherwise $d_{a}(p, q)=\|\varphi\|_{\infty}$, in the notation of $\S 1$.

We now specialize to consideration of a fixed component $C_{\alpha, \beta}$. Because $\lim _{\varepsilon \rightarrow 0^{+}} \frac{\sin \varepsilon}{\varepsilon}=1$, the same paths are rectifiable relative to $d_{a}$ and $d_{n}$ and the arc lengths are the same. Thus $\left(d_{a}\right)_{r}=\left(d_{n}\right)_{r}$, and we will write " $d_{r}$ " for this metric. Similarly, $d_{a}^{\prime}=d_{n}^{\prime}$ and we write " $d^{\prime}$ " for this where no confusion would arise. Thus (assuming, as will later be proved, that $d_{r}$ is everywhere defined) $d_{r} \geq d^{\prime} \geq d_{a} \geq d_{n}$.

Proposition 5. If $p$ and $q$ are in $C_{\alpha, \beta}$ and either $\|p-q\|<1$ or $\|p-q\|=1$ and $\operatorname{rank}(p \wedge(1-q))=\operatorname{rank}(q \wedge(1-p))$, then there is a path joining $p$ and $q$ of arc length $d_{a}(p, q)$. Hence in these cases $d_{r}(p, q)=d^{\prime}(p, q)=d_{a}(p, q)$, and a minimizing path exists.

Proof. Of course the hypothesis is stated redundantly, since $\|p-q\|<1$ implies $p \wedge(1-q)=q \wedge(1-p)=0$. Both cases are proved simultaneously by using the canonical representation of $(p, q)$. If $\|p-q\|=1$, we choose a unitary from $H_{01}$ onto $H_{10}$ and represent $H_{01} \oplus H_{10}$ in the same way as the generic part, with an angle of $\frac{\pi}{2}$. Then we can easily define a function $\gamma:\left[0, d_{a}(p, q)\right] \rightarrow C_{\alpha, \beta}$ such that $\gamma(0)=p, \gamma\left(d_{a}(p, q)\right)=q$, and $d_{a}(\gamma(s), \gamma(t))=|s-t|$. In informal language we define $\gamma$ by rotating $p$ toward $q$.

Explicitly, write $H \cong H_{00} \oplus\left(L^{2}\left(X^{\prime}\right) \oplus L^{2}\left(X^{\prime}\right)\right) \oplus\left(L^{2}(X) \oplus L^{2}(X)\right) \oplus H_{11}$ so that

and

$$
p=0 \oplus\left[\begin{array}{ll}
1 & 0 \\
0 & 0
\end{array}\right] \oplus\left[\begin{array}{ll}
1 & 0 \\
0 & 0
\end{array}\right] \oplus 1
$$

$$
q=0 \oplus\left[\begin{array}{ll}
0 & 0 \\
0 & 1
\end{array}\right] \oplus\left[\begin{array}{cc}
\cos ^{2} \varphi & \cos \varphi \sin \varphi \\
\cos \varphi \sin \varphi & \sin ^{2} \varphi
\end{array}\right] \oplus 1,
$$

where the second component (involving $L^{2}\left(X^{\prime}\right)$ ) is missing if $\|p-q\|<1$. Then

$$
\gamma(s)=0 \oplus\left[\begin{array}{cc}
\cos ^{2} s & \cos s \sin s \\
\cos s \sin s & \sin ^{2} s
\end{array}\right] \oplus\left[\begin{array}{cc}
\cos ^{2} \varphi_{s} & \cos \varphi_{s} \sin \varphi_{s} \\
\cos \varphi_{s} \sin \varphi_{s} & \sin ^{2} \varphi_{s}
\end{array}\right] \oplus 1,
$$

where $\varphi_{s}(x)=\min (s, \varphi(x))$. (Another choice would be to take $\varphi_{s}(x)=$ $s \varphi(x) / d_{a}(p, q)$. This choice would produce a smooth curve.)

Remark. If $\alpha$ or $\beta$ is finite, then $\operatorname{rank}(p \wedge(1-q))=\operatorname{rank}(q \wedge(1-p))$ for all $p, q$ in $C_{\alpha, \beta}$ and Proposition 5 is all-inclusive. Thus from now on the reader may as well assume that $\alpha$ and $\beta$ are infinite.

Proposition 6. If $p$ and $q$ are in $C_{\alpha, \beta}$, then there is a path of arc length at most $\pi$ joining $p$ and $q$. Hence $d_{r}(p, q) \leq \pi$.

Proof. Again we use the canonical representation of $(p, q)$. We construct a path $\gamma$ of length at most $\frac{\pi}{2}$ from $p$ to $r$, where $r$ is chosen so that $(r, q)$ satisfies the hypothesis of Proposition 5. There are two cases:

(i) $\operatorname{dim} H_{11} \leq \operatorname{dim} H_{00}$, 
(ii) $\operatorname{dim} H_{11}>\operatorname{dim} H_{00}$.

In both cases $\gamma(\cdot)$ is defined similarly to the proof of Proposition 5, except that now we rotate $p$ away from $q$, ending at an angle of $\frac{\pi}{2}$.

Explicitly, in case (i) we choose a unitary from $H_{11}$ onto a subspace $L$ of $H_{00}$. Then

$$
\begin{gathered}
H \cong\left(H_{00} \ominus L\right) \oplus H_{10} \oplus H_{01} \oplus(L \oplus L) \oplus\left(L^{2}(X) \oplus L^{2}(X)\right), \\
p=0 \oplus 1 \oplus 0 \oplus\left[\begin{array}{ll}
1 & 0 \\
0 & 0
\end{array}\right] \oplus\left[\begin{array}{ll}
1 & 0 \\
0 & 0
\end{array}\right], \\
q=0 \oplus 0 \oplus 1 \oplus\left[\begin{array}{ll}
1 & 0 \\
0 & 0
\end{array}\right] \oplus\left[\begin{array}{cc}
\cos ^{2} \varphi & \cos \varphi \sin \varphi \\
\cos \varphi \sin \varphi & \sin ^{2} \varphi
\end{array}\right] .
\end{gathered}
$$

We take

$$
r=0 \oplus 1 \oplus 0 \oplus\left[\begin{array}{ll}
0 & 0 \\
0 & 1
\end{array}\right] \oplus\left[\begin{array}{cc}
\cos ^{2}\left(\varphi-\frac{\pi}{2}\right) & \cos \left(\varphi-\frac{\pi}{2}\right) \sin \left(\varphi-\frac{\pi}{2}\right) \\
\cos \left(\varphi-\frac{\pi}{2}\right) \sin \left(\varphi-\frac{\pi}{2}\right) & \sin ^{2}\left(\varphi-\frac{\pi}{2}\right)
\end{array}\right] .
$$

In case (ii) we choose a unitary from a subspace $L$ of $H_{11}$ onto $H_{00}$. Then

$$
\begin{gathered}
H \cong H_{10} \oplus H_{01} \oplus(L \oplus L) \oplus\left(L^{2}(X) \oplus L^{2}(X)\right) \oplus\left(H_{11} \ominus L\right), \\
p=1 \oplus 0 \oplus\left[\begin{array}{ll}
1 & 0 \\
0 & 0
\end{array}\right] \oplus\left[\begin{array}{ll}
1 & 0 \\
0 & 0
\end{array}\right] \oplus 1, \\
q=0 \oplus 1 \oplus\left[\begin{array}{ll}
1 & 0 \\
0 & 0
\end{array}\right] \oplus\left[\begin{array}{cc}
\cos ^{2} \varphi & \cos \varphi \sin \varphi \\
\cos \varphi \sin \varphi & \sin ^{2} \varphi
\end{array}\right] \oplus 1
\end{gathered}
$$

We take

$$
r=1 \oplus 0 \oplus\left[\begin{array}{ll}
0 & 0 \\
0 & 1
\end{array}\right] \oplus\left[\begin{array}{cc}
\cos ^{2}\left(\varphi-\frac{\pi}{2}\right) & \cos \left(\varphi-\frac{\pi}{2}\right) \sin \left(\varphi-\frac{\pi}{2}\right) \\
\cos \left(\varphi-\frac{\pi}{2}\right) \sin \left(\varphi-\frac{\pi}{2}\right) & \sin ^{2}\left(\varphi-\frac{\pi}{2}\right)
\end{array}\right] \oplus 1
$$

In case (i) $r$ is orthogonal to $q$. Since $\operatorname{rank} r=\operatorname{rank} q=\alpha$, Proposition 5 applies to $(r, q)$. In case (ii) $r q=q r$ and $r \vee q=1$. Therefore

$$
\operatorname{rank}(r \wedge(1-q))=\operatorname{rank}(1-q)=\beta=\operatorname{rank}(1-r)=\operatorname{rank}(q \wedge(1-r)) .
$$

Thus again Proposition 5 applies. To complete the proof, we join $r$ to $q$ by a path of arc length $\frac{\pi}{2}$.

Lemma 7. Assume $p$ and $q$ are in $C_{\alpha, \beta}$ and $\operatorname{rank}(p-p \wedge q) \neq \operatorname{rank}(q-p \wedge q)$. Then $d^{\prime}(p, q) \geq \pi$.

Proof. If $d^{\prime}(p, q)=d_{a}^{\prime}(p, q)<\pi$, then there is an $r$ such that $d_{a}(p, r)<\frac{\pi}{2}$ and $d_{a}(r, q)<\frac{\pi}{2}$. Denote the ranges of $p, r$, and $q$ by $L, M$, and $N$, respectively. Let $M_{0}=r(L \cap N)$. Since $\|p-r\|<1$, the restriction of $r$ to $L$ is a topological isomorphism from $L$ onto $M$. Thus $L / L \cap N$ is topologically isomorphic to $M / M_{0}$. It follows that $\operatorname{rank}(p-p \wedge q)=\operatorname{dim}(L \ominus L \cap N)=$ $\operatorname{dim}\left(M \ominus M_{0}\right)$. Similarly, $\operatorname{rank}(q-p \wedge q)=\operatorname{dim}\left(M \ominus M_{0}\right)$. This contradiction completes the proof.

Remark. Lemma 7 applies in particular if $p$ is a proper subprojection of $q$.

We are now ready to "calculate" $d_{r}$ and show that $d_{r}=d^{\prime}$. For the sake of clarity we describe the notation before stating a formal theorem. Assume that $p$ and $q$ are in $C_{\alpha, \beta}$ and $\operatorname{rank}(p \wedge(1-q)) \neq \operatorname{rank}(q \wedge(1-p))$. Let $M$ and $N$ be the ranges of $p$ and $q$ and refer to the canonical representation of $(p, q)$. 
For $0 \leq \theta<\frac{\pi}{2}$, let $E_{\theta}=\{x \in X: \varphi(x) \geq \theta\}$ and let $H_{\theta}$ be the closed subspace of $H_{0}$ corresponding to the projection

$$
\left[\begin{array}{cc}
\chi_{E_{\theta}} & 0 \\
0 & \chi_{E_{\theta}}
\end{array}\right], \quad \text { where } \chi_{E_{\theta}}(x)= \begin{cases}1, & x \in E_{\theta}, \\
0, & x \notin E_{\theta},\end{cases}
$$

and the matrix has the same meaning as in $\S 1$. Let $M_{\theta}=M \cap H_{\theta}$ and $N_{\theta}=$ $N \cap H_{\theta}$. (Thus $M_{0}$ is the entire generic part of $M$.)

It may be that for some $\theta$ in $\left(0, \frac{\pi}{2}\right) \operatorname{dim}\left(H_{10} \oplus M_{\theta}\right)=\operatorname{dim}\left(H_{01} \oplus N_{\theta}\right)$. (Note that $\operatorname{dim} M_{\theta}=\operatorname{dim} N_{\theta}$.) If this occurs let $\theta_{0}$ be the supremum of all $\theta<\frac{\pi}{2}$ such that $\operatorname{dim}\left(H_{10} \oplus M_{\theta}\right)=\operatorname{dim}\left(H_{01} \oplus N_{\theta}\right)$. (Thus $\theta_{0}$ may be $\frac{\pi}{2}$.) If this does not occur, let $\theta_{0}=0$.

We remark that in the separable case there is a more suggestive way to describe $\theta_{0}$ : We have $\operatorname{dim}\left(H_{10} \oplus M_{\theta}\right)=\operatorname{dim}\left(H_{01} \oplus N_{\theta}\right)$ if and only if $M_{\theta}$ (or equivalently $H_{\theta}$ ) is infinite dimensional. Then the lowest point in the essential spectrum of the restriction of $p q p$ to its initial space is $\cos ^{2} \theta_{0}$. (The initial space of $p q p$ is $M_{0} \oplus H_{11}$.) Furthermore if $H_{10}$ is finite dimensional, $\cos ^{2} \theta_{0}$ is also the lowest point in the essential spectrum of $\left.p q p\right|_{M}$. Since $\operatorname{rank}(p \wedge(1-q)) \neq \operatorname{rank}(q \wedge(1-p))$, at least one of $H_{10}, H_{01}$ is finite dimensional. Thus $\theta_{0}$ can be calculated from the images of $p$ and $q$ in the Calkin algebra. In particular $\theta_{0}=0$ if and only if the Calkin image of $p$ is a subprojection (not necessarily proper) of the Calkin image of $q$, or vice-versa. Note that the hypothesis rank $(p \wedge(1-q)) \neq \operatorname{rank}(q \wedge(1-p))$ cannot be verified in terms of the Calkin images of $p$ and $q$.

Theorem 8. If $p$ and $q$ are in $C_{\alpha, \beta}$ and $\operatorname{rank}(p \wedge(1-q)) \neq \operatorname{rank}(q \wedge(1-p))$, then $d_{r}(p, q)=d^{\prime}(p, q)=\pi-\theta_{0}$.

Proof. 1. $d_{r}(p, q) \leq \pi-\theta_{0}$ :

In view of Proposition 6, we may assume $\theta_{0}>0$. Choose $\theta$ such that $0<\theta<\theta_{0}$. Then $\operatorname{dim}\left(H_{10} \oplus M_{\theta}\right)=\operatorname{dim}\left(H_{01} \oplus N_{\theta}\right)$. We can find a path of length at most $\frac{\pi}{2}-\theta$ joining $p$ to $r$, where the $H_{\theta}$-component of $r$ has range $H_{\theta} \ominus N_{\theta}$, and the other components of $r$ are the same as those of $p$ (cf. proof of Proposition 6). Then $\operatorname{rank}(r \wedge(1-q))=\operatorname{rank}(q \wedge(1-r))=\operatorname{dim}\left(H_{01} \oplus N_{\theta}\right)$. Thus Proposition 5 implies $d_{r}(r, q) \leq \frac{\pi}{2}$, and hence $d_{r}(p, q) \leq \pi-\theta$. Since $\theta$ can be taken arbitrarily close to $\theta_{0}, d_{r}(p, q) \leq \pi-\theta_{0}$.

2. $d^{\prime}(p, q) \geq \pi-\theta_{0}$ :

We already know $d^{\prime}(p, q) \geq d_{a}(p, q)=\frac{\pi}{2}$. Thus we may assume $\theta_{0}<\frac{\pi}{2}$. Choose $\theta$ such that $\theta_{0}<\theta<\frac{\pi}{2}$. Then $\operatorname{dim}\left(H_{10} \oplus M_{\theta}\right) \neq \operatorname{dim}\left(H_{01} \oplus N_{\theta}\right)$. We can find a path of length at most $\theta$ joining $p$ to $r$, where the $\left(H_{0} \ominus H_{\theta}\right)$ component of $r$ is the same as that of $q$ and the other components of $r$ are the same as those of $p$. Then $\operatorname{rank}(r-r \wedge q)=\operatorname{dim}\left(H_{10} \oplus M_{\theta}\right) \neq \operatorname{dim}\left(H_{01} \oplus N_{\theta}\right)=$ $\operatorname{rank}(q-r \wedge q)$. Thus Lemma 7 implies $d^{\prime}(r, q) \geq \pi$. Then $d^{\prime}(p, q) \geq$ $d^{\prime}(r, q)-d^{\prime}(p, r) \geq d^{\prime}(r, q)-d_{r}(p, r) \geq \pi-\theta$. Since $\theta$ can be taken arbitrarily close to $\theta_{0}, d^{\prime}(p, q) \geq \pi-\theta_{0}$.

Remark. On the basis of what has already been said, it is obvious that when $\alpha$ and $\beta$ are infinite, there exist examples of $(p, q)$ satisfying the hypotheses of Theorem 8 for any $\theta_{0}$ in $\left[0, \frac{\pi}{2}\right]$ (even if $H$ is not separable).

All that remains is to settle the question of existence of minimizing paths. By Propositions 5 and 6, minimizing paths always exist if $d_{r}(p, q)<\frac{\pi}{2}$ or if $d_{r}(p, q)=\pi$. 
Theorem 9. If $\operatorname{rank}(p \wedge(1-q)) \neq \operatorname{rank}(q \wedge(1-p))$ and if $d_{r}(p, q)=\frac{\pi}{2}$, then $p$ and $q$ are not joined by a minimizing path.

Proof. If a minimizing path exists, then there is an $r$ such that $d_{a}(p, r)=$ $d_{a}(r, q)=\frac{\pi}{4}$. Let $L, M, N$ be the ranges of $p, r, q$, respectively. Referring to the canonical representation of $(p, r)$, we can find a unitary $U$ from $L$ onto $M$ with the following properties:

1. $(U x, x) \geq \cos \frac{\pi}{4}=1 / \sqrt{2}$, for every unit vector $x$ in $L$. (In particular $(U x, x) \geq 0$.)

2. If $(U x, x)=\cos \frac{\pi}{4}$ then $r x=\cos \frac{\pi}{4} U x$ and $p U x=\cos \frac{\pi}{4} x$.

$U$ is closely related to Dixmier's " $S_{V_{0}}$ " in [6]. In fact the $H_{0}$-component of $U$ is just the restriction of $S_{V_{0}}$ to $L_{0}$. (The $H_{11}$-component of $U$ is the identity.) We also find a unitary $V$ from $M$ onto $N$ with similar properties.

Then $V U$ is a unitary from $L$ onto $N$. Without loss of generality, we assume

$$
\operatorname{dim}\left(L \cap N^{\perp}\right)=\operatorname{rank}(p \wedge(1-q))>\operatorname{rank}(q \wedge(1-p))=\operatorname{dim}\left(N \cap L^{\perp}\right) .
$$

Thus there is a unit vector $x$ in $L \cap N^{\perp}$ such that $V U x$ is orthogonal to $N \cap L^{\perp}$. Let $U x=y$ and $V U x=z$. Since $x$ is in $N^{\perp}$ and $z$ is in $N$, $x \perp z$. By property 1 for $U$ and $V,(y, x) \geq 1 / \sqrt{2}$ and $(y, z) \geq 1 / \sqrt{2}$. Since $\|y\|=1$, this implies $y=(1 / \sqrt{2}) x+(1 / \sqrt{2}) z$. Then property 2 for $U$ applies, and we conclude that $p y=(1 / \sqrt{2}) x$. Since $p y=(1 / \sqrt{2}) x+(1 / \sqrt{2}) p z$, this implies $p z=0$. In other words, $z$ is in $N \cap L^{\perp}$, a contradiction.

The remainder of this section could be simplified significantly in the case where $H$ is separable. The reader who wishes to assume $H$ separable may substitute his own simpler arguments in appropriate places. We have a reason for presenting the material in this way, which is explained by Remark 3 in the next section.

Lemma 10. Let $h$ be a positive selfadjoint operator on a Hilbert space $M_{0}$ and $\lambda$ a cardinal number such that $\lambda \leq \operatorname{rank} E_{(0, \varepsilon)}(h), \forall \varepsilon>0$, where $E_{(0, \varepsilon)}(h)$ is the spectral projection of $h$ for the interval $(0, \varepsilon)$. Then there is a closed subspace $M_{1}$ such that $\operatorname{dim} M_{1}=\lambda$ and $M_{1}$ is disjoint from the range of $h$.

Sublemma. There are a decreasing sequence $\left(\varepsilon_{n}\right)$ of positive numbers and a nondecreasing sequence $\left(\lambda_{n}\right)$ of cardinal numbers such that $\varepsilon_{n} \rightarrow 0, \sup _{n} \lambda_{n}=\lambda$, and $\lambda_{n} \leq \operatorname{rank} E_{\left(\varepsilon_{n+1}, \varepsilon_{n}\right)}(h), \forall n$.

Proof. We consider two cases:

(i) $\lambda$ is the supremum of countably many cardinal numbers, each strictly less than $\lambda$.

(ii) $\lambda$ is not such a supremum.

In case (i) we choose an increasing sequence $\left(\lambda_{n}\right)$ such that $\sup _{n} \lambda_{n}=\lambda$ and $\lambda_{n}<\lambda, \forall n$. In case (ii) we take $\lambda_{n}=\lambda, \forall n$.

Now we can construct the $\varepsilon_{n}$ 's recursively, starting with $\varepsilon_{1}=1$. If $\varepsilon_{n}$ has already been chosen, we will choose $\varepsilon_{n+1}$ such that $0<\varepsilon_{n+1} \leq \min \left(\frac{1}{n+1}, \varepsilon_{n}\right)$ and $\operatorname{rank} E_{\left(\varepsilon_{n+1}, \varepsilon_{n}\right)}(h) \geq \lambda_{n}$. This is possible because $\sup _{m}\left(\operatorname{rank} E_{\left(\varepsilon_{n} / m, \varepsilon_{n}\right)}(h)\right) \geq$ $\lambda$. In case (i), it is clearly impossible that $\operatorname{rank} E_{\left(\varepsilon_{n} / m, \varepsilon_{n}\right)}(h) \leq \lambda_{n}, \forall m$. In case (ii), clearly $\operatorname{rank} E_{\left(\varepsilon_{n} / m, \varepsilon_{n}\right)}(h) \geq \lambda$ for some $m$. 
Proof of Lemma 10. Choose a sequence $\left(c_{n}\right)$ of positive numbers such that $\sum_{1}^{\infty} c_{n}^{2}=1$ and $\sum_{1}^{\infty}\left(c_{n}^{2} / \varepsilon_{n}^{2}\right)=\infty$. Choose a Hilbert space $L$ and a nondecreasing sequence of closed subspaces $L_{n}$ such that $\sup _{n} L_{n}=L, \operatorname{dim} L=\lambda$, and $\operatorname{dim} L_{n}=\lambda_{n}$. Let $P_{n}$ be the projection operator on $L$ with range $L_{n}$. For each $n$ choose a partial isometry $U_{n}$ such that $U_{n}^{*} U_{n}=P_{n}$ and $U_{n} U_{n}^{*} \leq E_{\left(\varepsilon_{n+1}, \varepsilon_{n}\right)}(h)$. Let $V_{n}=U_{n} \sum_{1}^{n} c_{n-k+1}\left(P_{k}-P_{k-1}\right)$, where $P_{0}=0$. We can define an isometry $V: L \rightarrow M_{0}$ by $V=\sum_{1}^{\infty} V_{n}$, where the sum converges strongly. Since the $V_{n}$ 's have mutually orthogonal ranges, $V^{*} V=\sum_{1}^{\infty} V_{n}^{*} V_{n}$, and it is easy to check that $V$ really is an isometry and that the sum really converges. Now we let $M_{1}$ be the range of $V$, and we need only show that $V x$ is not in the range of $h$, for a nonzero $x$ in $L$. But clearly $V x$ in range $h$ implies $\sum_{1}^{\infty}\left(\left\|V_{n} x\right\|^{2} / \varepsilon_{n}^{2}\right)<\infty$.

$$
\begin{aligned}
\frac{\left\|V_{n} x\right\|^{2}}{\varepsilon_{n}^{2}} & =\frac{1}{\varepsilon_{n}^{2}} \sum_{1}^{n} c_{n-k+1}^{2}\left(\left(P_{k}-P_{k-1}\right) x, x\right) \\
& \geq \sum_{1}^{n} \frac{c_{n-k+1}^{2}}{\varepsilon_{n-k+1}^{2}}\left(\left(P_{k}-P_{k-1}\right) x, x\right) .
\end{aligned}
$$

Thus

$$
\sum_{1}^{\infty} \frac{\left\|V_{n} x\right\|^{2}}{\varepsilon_{n}^{2}} \geq \sum_{k=1}^{\infty}\left(\sum_{n \geq k} \frac{c_{n-k+1}^{2}}{\varepsilon_{n-k+1}^{2}}\right)\left(\left(P_{k}-P_{k-1}\right) x, x\right)
$$

The last sum is $\infty$, since $\left(\left(P_{k}-P_{k-1}\right) x, x\right)>0$ for some $k$ and

$$
\sum_{n \geq k} \geq \frac{c_{n-k+1}^{2}}{\varepsilon_{n-k+1}^{2}}=\sum_{n=1}^{\infty} \frac{c_{n}^{2}}{\varepsilon_{n}^{2}}=\infty
$$

Lemma 11. Let $L$ and $M_{0}$ be Hilbert spaces, $t$ a positive number, and $S$ a positive invertible selfadjoint operator on $M_{0}$. Assume that $\|S\|=t$, that $S$ does not achieve its norm, and that $\operatorname{dim} L \leq \operatorname{rank} E_{(t-\varepsilon, t)}(S), \forall \varepsilon>0$. Then there is an operator $T: L \oplus M_{0} \rightarrow M_{0}$ such that $\|T\| \leq \frac{1}{t}$ and $q: \operatorname{graph} T \rightarrow \operatorname{graph} S$ is one-to-one with dense range, where $q$ is the projection operator on $L \oplus M_{0} \oplus M_{0}$ whose range is graph $S$. (Thus $\left.q\right|_{L}=0$.)

Remark. The hypothesis that $S$ be positive is given only to simplify the notation. We could equally well work with the spectral projections of $|S|$ instead of $S$.

Proof. $\left.q\right|_{M_{0} \oplus M_{0}}$ is given by the operator matrix

$$
\left(\begin{array}{cc}
\left(1+S^{2}\right)^{-1} & \left(1+S^{2}\right)^{-1} S \\
S\left(1+S^{2}\right)^{-1} & S\left(1+S^{2}\right)^{-1} S
\end{array}\right)
$$

$T$ will be given by a pair $\left(T^{\prime}, T_{0}\right)$, where $T^{\prime}: L \rightarrow M_{0}$ and $T_{0}: M_{0} \rightarrow M_{0}$. Then $\|T\| \leq \frac{1}{t}$ is equivalent to $T_{0} T_{0}^{*}+T^{\prime} T^{*} \leq 1 / t^{2}$. Since graph $T$ and graph $S$ are topologically isomorphic to $L \oplus M_{0}$ and $M_{0}$, respectively, instead of proving that $q$ is one-to-one with dense range between the graphs, we may instead prove that the appropriate operator $Q: L \oplus M_{0} \rightarrow M_{0}$ is one-to-one with dense range. So $Q$ is given by the pair $\left(Q^{\prime}, Q_{0}\right)$, where $Q^{\prime}=\left(1+S^{2}\right)^{-1} S T^{\prime}$ and $Q_{0}=\left(1+S^{2}\right)^{-1}\left(1+S T_{0}\right)$. We may further simplify the problem by replacing $Q$ with $S^{-1}\left(1+S^{2}\right) Q$, which is given by the pair $\left(T^{\prime}, S^{-1}+T_{0}\right)$. 
Now we apply Lemma 10 with $h=S^{-1}-\frac{1}{t}$ and $\lambda=\operatorname{dim} L$. Let $P$ be the projection operator on $M_{0}$ whose range is the space $M_{1}$ of Lemma 10, let $T^{\prime}$ be a one-to-one operator such that $T^{\prime} T^{\prime *}=P / t^{2}$, and let $T_{0}=\frac{1}{t}(-1+P)$. Then $T_{0} T_{0}^{*}+T^{\prime} T^{*}=1 / t^{2}$ so that $\|T\| \leq \frac{1}{t}$. Since $S^{-1}+T_{0}=h+\frac{1}{t} P \geq h$, and since $h$ is one-to-one (because $S$ does not achieve its norm), $S^{-1}+T_{0}$ is one-to-one with dense range. Thus we need only show that the ranges of $T^{\prime}$ and $S^{-1}+T_{0}$ are disjoint. But if $T^{\prime} x=\left(S^{-1}+T_{0}\right) y=h y+\frac{1}{t} P y$, then $h y=T^{\prime} x-\frac{1}{t} P y$, an element of $M_{1}$. Since $M_{1}$ is disjoint from the range of $h$, this implies $y=0$. Q.E.D.

Theorem 12. If $p$ and $q$ are in $C_{\alpha, \beta}$ and $\frac{\pi}{2}<d_{r}(p, q)<\pi$, then there is a minimizing path joining $p$ and $q$. Thus to summarize: A minimizing path joining $p$ and $q$ always exists if $d_{r}(p, q) \neq \frac{\pi}{2}$; and if $d_{r}(p, q)=\frac{\pi}{2}, a$ minimizing path exists if and only if $\operatorname{rank}(p \wedge(1-q))=\operatorname{rank}(q \wedge(1-p))$.

Proof. We may assume $\operatorname{rank}(p \wedge(1-q))>\operatorname{rank}(q \wedge(1-p))$. Choose a unitary from $H_{01}$ onto a subspace $L_{1}$ of $H_{10}$, and let $L=H_{10} \ominus L_{1}$. Let $\theta_{0}, M, N$ be as in Theorem 8, so that $d_{r}(p, q)=\pi-\theta_{0}$. It may be that $\operatorname{dim}\left(H_{10} \oplus M_{\theta_{0}}\right)$ $=\operatorname{dim}\left(H_{01} \oplus N_{\theta_{0}}\right)$. If so, the method of point 1 of the proof of Theorem 8 produces the desired minimizing path. Thus we assume $\operatorname{dim}\left(H_{10} \oplus M_{\theta_{0}}\right)>$ $\operatorname{dim}\left(H_{01} \oplus N_{\theta_{0}}\right)$. Then for any $\theta$ such that $0<\theta<\theta_{0}$ we have

$$
\operatorname{dim}\left(L \oplus\left(M_{\theta} \ominus M_{\theta_{0}}\right)\right)=\operatorname{dim}\left(N_{\theta} \ominus N_{\theta_{0}}\right) .
$$

(This is an easy exercise in cardinal number theory.) Now choose $\theta_{1}$ such that $0<\theta_{1}<\theta_{0}$, and let $H_{1}=L \oplus\left(H_{\theta_{1}} \ominus H_{\theta_{0}}\right)$. Then $H_{1}$ is a reducing subspace for both $p$ and $q$. It is another easy exercise to show that both the $H_{1}$ - and $H_{1}^{\perp}$-components of $p$ and $q$ still agree in rank and co-rank. Since the $H_{1}^{\perp}$-components clearly satisfy the hypothesis of Proposition 5, we may change notation and assume $H=H_{1}$. (In the new notation what was $M_{\theta_{1}} \ominus M_{\theta_{0}}$ becomes, simply, $M_{0}$.)

Now we can identify $H_{0} \ominus M_{0}$ with $M_{0}$ so that $N$ is the graph of a positive operator $S: M_{0} \rightarrow M_{0}$. In terms of the notation for the canonical representation given in $\S 1, S$ is just the multiplication operator for $\tan \varphi$. Then $\|S\|=\tan \theta_{0}, S$ does not achieve its norm, and $S$ is bounded below by $\tan \theta_{1}$. Since $\operatorname{dim}\left(L \oplus M_{\theta}\right)=\operatorname{dim} N_{\theta}, \forall \theta \in\left(\theta_{1}, \theta_{0}\right)$, we have $\operatorname{dim} L \leq$ rank $E_{\left(\tan \theta_{0}-\varepsilon, \tan \theta_{0}\right)}(S), \forall \varepsilon>0$. Thus Lemma 11 applies with $t=\tan \theta_{0}$.

Let $r$ be the projection whose range is graph $T$. Since $\|T\| \leq \frac{1}{t}, d_{a}(p, r) \leq$ $\tan ^{-1}\left(\frac{1}{t}\right)=\frac{\pi}{2}-\theta_{0}<\frac{\pi}{2}$. Therefore there is a path of length at most $\frac{\pi}{2}-\theta_{0}$ joining $p$ to $r$. Because the projection from graph $T$ to graph $S$ is one-to-one with dense range, $r \wedge(1-q)=q \wedge(1-r)=0$. Therefore, by Proposition 5, there is a path of length at most $\frac{\pi}{2}$ joining $r$ to $q$. Combining the two paths gives the desired minimizing path.

\section{CONCLUDING REMARKS}

1. The metric $d_{r}$ is not defined on all of $\mathscr{P}$ but only on each $C_{\alpha, \beta}$. We can easily extend $d_{r}$ to a metric on $\mathscr{P}$. Just choose any number $\delta>\pi$ and define $d_{r}(p, q)=\delta$ whenever $p$ and $q$ are in different components of $\mathscr{P}$. The most natural choice would be $\delta=\infty$, but this is not allowed. The extended $d_{r}$ is 
rectifiable if we redefine "rectifiable" to mean "the restriction to each component is rectifiable in the original sense".

2. It is obvious that minimizing paths in $C_{\alpha, \beta}$ are usually not unique. For completeness we state without proof the facts on uniqueness. For $p \neq q, p$ and $q$ are joined by a unique minimizing path if and only if $d_{a}(p, q)<\frac{\pi}{2}$, the function $\varphi$ appearing in the canonical representation is essentially constant, and either $H_{11}=0$ or $H_{00}=0$.

3. We can generalize this paper by considering the set of projections in a von Neumann algebra $M$, instead of the set of projections on $H$. There are some obvious changes which have to be made. For example, $\alpha$ and $\beta$ now have to be Murray-von Neumann equivalence classes of projections in $M$ instead of cardinal numbers. The often-stated property " $\operatorname{rank}(p \wedge(1-q))=$ $\operatorname{rank}(q \wedge(1-p))$ " becomes " $p \wedge(1-q) \sim q \wedge(1-p)$ ". The hardest result to generalize to this context is Lemma 10. The new version of this replaces $\lambda$ with a projection $r$ such that $r$ is equivalent to some subprojection of $E_{(0, \varepsilon)}(h)$ for each $\varepsilon>0$. The conclusion is that there is a projection $r_{1}$ such that $r_{1} \sim r$, $r_{1} \leq E_{(0, \infty)}(h)$, and the range of $r_{1}$ is disjoint from the range of $h$. It is just a somewhat tedious exercise to prove this generalization, using the given proof of Lemma 10 as a model, though the generalized version does present some additional complications.

4. The basic results of this paper, including especially Theorem 9, can be used to prove a sort of metric index zero theorem: If $p, q$, and $r$ are distinct projections such that

$$
\sin ^{-1}(\|p-r\|)+\sin ^{-1}(\|r-q\|) \leq \frac{\pi}{2},
$$

then $\operatorname{rank}(p \wedge(1-q))=\operatorname{rank}(q \wedge(1-p))$.

5. The often-stated property “ $\operatorname{rank}(p \wedge(1-q))=\operatorname{rank}(q \wedge(1-p))$ " was used by Davis in [4], where $p$ and $q$ are said to be "equivalently positioned". The concept of direct rotation from [4] and Propositions 3.1 and 3.2 of Davis and Kahane [5] probably have some relation to the underlying ideas of some of our proofs, including our proof of Proposition 5. Sano and Watatani [15] have applied angles between projections in the context of subfactors. Several authors have previously considered rectifiable metrics (also called Finsler metrics) for various spaces of operators from a differential geometric point of view. Atkin $[1,2]$ considered various groups of invertible operators instead of $\mathscr{P}$ and Wilkins [17] considered Grassmannians of $C^{*}$-algebras. Various works of Salinas, Corach, Porta, and Recht have some overlap with Proposition 5 above and implicitly also Corollary 4 , and we proceeded to give more detail. In the works of these authors a different metric is used, so that all of their arc lengths are exactly the doubles of ours. Also these authors consider the set of projections in an arbitrary $C^{*}$-algebra, not necessarily a von Neumann algebra as in this paper. Thus their results similar to Proposition 5 above are more general, but they do not have results analogous to Theorems 8, 9, and 12 above, which are not valid in the more general context. Porta and Recht [11] and Salinas [14] have results which include the case of Proposition 5 where $\|p-q\|<1$. This could be used to prove that $d_{a}$ is a metric. Corach, Porta, and Recht [3] consider the space of not necessarily selfadjoint idempotents in a $C^{*}$-algebra. We also note that the answer to the question in Remark 2.22(b) of [14] is "no". (See the proof of Proposition 5 above and especially the last sentence.) 


\section{REFERENCES}

1. C. J. Atkin, The Finsler geometry of groups of isometries of Hilbert space, J. Austral. Math. Soc. 42 (1987), 196-222.

2. _ The Finsler geometry of certain covering groups of operator groups, Hokkaido Math. J. 18 (1989), 45-77.

3. G. Corach, H. Porta and L. Recht, The geometry of spaces of projections in $C^{*}$-algebras, Adv. in Math. (to appear).

4. C. Davis, Separation of two linear subspaces, Acta Sci. Math. (Szeged) 19 (1958), 172-187.

5. C. Davis and W. M. Kahane, The rotation of eigenvalues by a perturbation. III, SIAM J. Numer. Anal. 7 (1970), 1-46.

6. J. Dixmier, Position relative de deux variétés linéaires fermées dans un espace de Hilbert, Rev. Sci. 86 (1948), 387-399.

7. M. Gromov, Structure métriques pour les variétés riemanniennes, CEDIC, Paris, 1981.

8. P. Halmos, Two subspaces, Trans. Amer. Math. Soc. 144 (1969), 381-389.

9. M. G. Kreĭn, M. A. Krasnosel'skii, and D. P. Mil'man, Defect numbers of linear operators in Banach space and some geometrical problems, Sbor. Trudov Inst. Mat. Akad. Nauk SSSR, no. 11, 1948, pp. 97-112. (Russian)

10. G. Pedersen, Measure theory for $C^{*}$-algebras. II, Math. Scand. 22 (1968), 63-74.

11. H. Porta and L. Recht, Minimality of geodesics in Grassmann manifolds, Proc. Amer. Math. Soc. 100 (1987), 464-466.

12. I. Raeburn and A. Sinclair, The $C^{*}$-algebra generated by two projections, Math. Scand. 65 (1989), 278-290.

13. W. Rinow, Die innere geometrie der metrischen räume, Springer-Verlag, Berlin, Göttingen, and Heidelberg, 1961.

14. N. Salinas, The Grassmann manifold of a $C^{*}$-algebra and Hermitian holomorphic bundles, Operator Theory: Adv. Appl. 28 (1988), 267-289.

15. T. Sano and Y. Watatani, Angles between two subfactors, preprint.

16. V. S. Sunder, $N$ subspaces, Canad. J. Math. 40 (1988), 38-54.

17. D. R. Wilkins, The Grassmann manifold of a $C^{*}$-algebra, Proc. Roy. Irish Acad. Sect A 90 (1990), 99-116.

Department of Mathematics, Purdue University, West Lafayette, Indiana 47907

E-mail address: lgb@math.purdue.edu 